\title{
TESTE DE CONDUTIVIDADE ELÉTRICA EM SEMENTES DE Albizia hassleri (Chodat) Burkart. FABACEAE-MIMOSOIDEAE ${ }^{1}$
}

\author{
José Luis Soto Gonzales², Rinaldo César de Paula ${ }^{3}$ e Sérgio Valiengo Valeri
}

\begin{abstract}
RESUMO - Este trabalho teve por objetivo verificar o uso do teste de condutividade elétrica para avaliar a qualidade fisiológica de sementes de Albizia hassleri (Chod) Burkart. provenientes de diferentes matrizes. O teste de condutividade elétrica foi realizado a $25^{\circ} \mathrm{C}$, com cinco repetições de 20 sementes embebidas em $75 \mathrm{~mL}$ de água destilada por $2,4,6,12,24,48,72,96$ e $120 \mathrm{~h}$. O teste de germinação foi conduzido a 25 ${ }^{\circ} \mathrm{C}$ com cinco repetições de 20 sementes por 19 dias. Os dados foram submetidos à análise de variância e as médias de matrizes, comparadas pelo teste de Scott-Knott a 5\% de probabilidade. Adicionalmente, procedeuse ao estudo de correlações entre os resultados de condutividade elétrica com os do teste de germinação e ao ajuste de regressões entre os valores de sementes com protrusão de radícula e de plântulas normais em função dos valores de condutividade elétrica, em cada período de embebição das sementes. Verificou-se grande variabilidade entre as matrizes, tanto no teste de germinação quanto no de condutividade elétrica. Os resultados de condutividade elétrica apontaram baixas estimativas de correlações com os obtidos no teste de germinação. As equações de regressão apresentaram baixos valores de coeficiente de determinação, denotando-se a grande variabilidade dos resultados. Pode-se concluir que o teste de condutividade elétrica não foi adequado para discriminação da qualidade fisiológica de sementes de A. hasslerii provenientes de diferentes árvores-matriz.
\end{abstract}

Palavras-chave: Vigor, deterioração e qualidade de sementes florestais.

\section{ELECTRICAL CONDUCTIVITY TEST IN Albizia hassleri (Chodat) Burkart. FABACEAE-MIMOSOIDEAE SEEDS}

\begin{abstract}
The objective of this research was to study the electrical conductivity test to evaluate the physiological quality of Albizia hassleri (Chod) Burkart. seeds. Sixteen mother tree plants were used and the conductivity test was performed at $25^{\circ} \mathrm{C}$ with the use of $75 \mathrm{~mL}$ of distilled water in periods of 2, 4, 6, 12, 24, 48, 72, 96 and 120 hours, with five replications of 20 seeds. The germination test was carried out at $25{ }^{\circ} \mathrm{C}$, and the final count was made on the 19th day. A completely randomized experimental design was used and the data were submitted to the variance analysis and the Scott-Knott test at $5 \%$ of probability to compare the mother tree means. Besides, correlations were performed between the electrical conductivity results and the germination percentage and normal seedlings and also the regression between both variables. A high variability was verified among the mother trees, both in the germination and in the electrical conductivity test. The results showed low correlation between the germination percentage and electrical conductivity. The regression between the electrical conductivity and germination presented a low determination coefficient. These data demonstrated that the electrical conductivity test was not adequate to discriminate the physiological seed quality of $\boldsymbol{A}$. hasslerii obtained from different mother trees.
\end{abstract}

Keywords: : Vigor, deterioration, tree seeds quality.

\footnotetext{
${ }^{1}$ Recebido em 18.09.2007 e aceito para publicação em 29.05.2009.

${ }^{2}$ Programa de Pós-Graduação em Agronomia (Produção Vegetal) da Universidade Estadual Paulista Júlio de Mesquita Filho (UNESP). E-mail: <jose.gonzales@ posgrad.fcav.unesp.br>.

${ }^{3}$ Departamento de Produção Vegetal da UNESP. E-mail:<valeri@fcav.unesp.br>e <rcpaula@ fcav.unesp.br>.
} 


\section{INTRODUÇÃO}

Albizia hassleri (Chod) Burkart, popularmente conhecida como farinha-seca, pertence à família Fabaceae-Mimosoideae. É uma espécie arbórea madeireira, podendo atingir cerca de $20 \mathrm{~m}$ de altura e $60 \mathrm{~cm}$ de diâmetro, sendo característica de matas, principalmente na Floresta Atlântica. A farinha-seca produz, anualmente, grande quantidade de sementes, que devem ser colocadas para germinar tão logo sejam colhidas, devido à sua curta viabilidade (LORENZI, 1992).

O teste de condutividade elétrica (CE) baseia-se no princípio de que, com o processo de deterioração, ocorre a lixiviação dos constituintes celulares das sementes embebidas em água devido à perda da integridade dos sistemas de membranas celulares. Desse modo, baixa condutividade significa alta qualidade da semente, e alta condutividade sugere o menor vigor desta, ou seja, maior saída de lixiviados da semente(VIEIRAe KRZYZANOWSKI, 1999). De acordo com Loeffler et al. (1988), os valores de CE estão associados ao estado fisiológico das sementes e aos níveis de germinação de cada espécie.

Fundamentado na integridade dos sistemas de membranas, o teste de condutividade elétrica é de ampla importância na determinação de vigor de sementes, em virtude de possibilitar que o processo de deterioração seja detectado em sua etapa inicial, permitindo que os efeitos na qualidade fisiológica das sementes sejam reduzidos ou minimizados (DIAS e MARCOS FILHO, 1995).

O teste apresenta uma série de características interessantes para uso na estimativa do vigor de sementes entre diferentes lotes, como a rapidez, objetividade, baixo custo e possuir base teórica consistente, sendo capaz de identificar a deterioração das sementes em seu estado inicial (AOSA, 1983; HAMPTON e TEKRONY, 1995).

Barbedo e Cícero (1998), trabalhando com Inga uruguensis, dividiram os lotes de sementes em três classes de germinação, associando-se a essas classes valores de condutividade elétrica.

O teste de condutividade elétrica foi eficiente para diferenciar lotes de sementes de Dalbergia nigra quando foram usadas 50 sementes embebidas em $75 \mathrm{~mL}$ de água deionizada a $25^{\circ} \mathrm{C}$, no período mínimo de $30 \mathrm{~h}$ (MARQUES et al., 2002a, b). Porém, o seu uso para avaliação da qualidade de sementes florestais é visto por Bonner (1998) com grandes ressalvas, dada a grande variabilidade genética normalmente presente nos lotes de sementes dessas espécies.

R. Árvore, Viçosa-MG, v.33, n.4, p.625-634, 2009
Ferraz et al. (1991) verificaram que o teste de condutividade elétrica não foi apropriado para avaliação de vigor das sementes de Carapa procera. Em sementes florestais, o uso do teste é bastante recente e vem sendo apontado como promissor para o monitoramento da qualidade fisiológica de lotes de sementes durante o armazenamento (SANTOS, 2004).

Dessa forma, este trabalho teve por objetivo verificar a possibilidade de aplicação do teste de condutividade elétrica para avaliar a qualidade fisiológica de sementes de farinha-seca provenientes de diferentes árvores matriz.

\section{MATERIAL E MÉTODOS}

Os frutos de farinha-seca (Albizia hassleri (Chod) (Burkart.)) foram coletados de 16 matrizes, selecionadas de acordo com critérios de Capelanes e Biella (1984), localizadas nas rodovias de acesso à cidade de Jaboticabal, SP, Brasil.

Os frutos foram colhidos diretamente das árvoresmatriz, com o auxílio de tesoura de poda alta, nos meses de outubro e novembro de 2005, quando apresentavam coloração castanha ou marrom, com base em Longhi (1995). Em seguida, foram acondicionados em sacos de plástico preto e transportados para o Viveiro Experimental de Plantas Ornamentais e Florestais da Faculdade de Ciências Agrárias e Veterinárias - FCAV, da Universidade Estadual Paulista - UNESP, Campus de Jaboticabal, SP. Os frutos foram, então, expostos ao sol para completarem a secagem e possibilitar a extração das sementes, a qual foi realizada manualmente, mantendo-se a identidade das matrizes.

Inicialmente, determinou-se o teor de água das sementes de cada matriz, pelo método da estufa a $105 \pm 3{ }^{\circ} \mathrm{C}$ por $24 \mathrm{~h}$ (BRASIL, 1992), usando-se duas repetições de 10 sementes.

No teste de (CE) foram usadas cinco repetições de 20 sementes por matriz, sendo essa quantidade usada em razão da sua pouca disponibilidade, as quais foram previamente pesadas em balança analítica com precisão de $0,001 \mathrm{~g}$. Cada repetição foi acondicionada em copo de plástico $(200 \mathrm{~mL})$ contendo $75 \mathrm{~mL}$ de água destilada, e, posteriormente, submetida a 2, 4, 6, 12, 24, 48, 72, 96 e 120 h de embebição a $25^{\circ} \mathrm{C}$. Decorrido cada período de embebição, efetuou-se a leitura de CE na solução de embebição das sementes usando um condutivímetro de bancada, marca Marconi, modelo CA-150, com 
constante 1,0. O valor de cada leitura de condutividade foi dividido pela respectiva massa da amostra, expressando-se os resultados de condutividade elétrica em $\mu \mathrm{S} \mathrm{cm}^{-1} \mathrm{~g}^{-1}$ de semente.

Os dados foram analisados segundo o delineamento inteiramente casualizado, individualmente para cada período de embebição, com 16 tratamentos (matrizes) e cinco repetições de 20 sementes. As médias de condutividade de cada matriz foram comparadas pelo teste de Scott-Knott a 5\% de probabilidade.

Após o teste de CE, foi conduzido o teste de germinação, usando-se cinco repetições de 20 sementes por matriz, de acordo com procedimento adotado por Tonin et al. (2005). O teste de germinação foi conduzido em caixas para germinação de plástico transparente e com tampa (gerbox), nas dimensões de 11 x 11 x 3 $\mathrm{cm}$. Como substrato, foram usadas duas folhas de papel Germitest, umedecidas com água em quantidade equivalente a duas vezes o peso do papel. $\mathrm{O}$ experimento foi conduzido em germinador vertical tipo BOD com temperatura constante de $25^{\circ} \mathrm{C}$ e fotoperíodo de $8 \mathrm{~h}$, por 19 dias. O número de sementes germinadas foi avaliado diariamente, adotando-se como critério de germinação a protrusão da radícula com pelo menos $2 \mathrm{~mm}$ de comprimento (DURAN e TORTOSA, 1985). Ao final do experimento, determinou-se a porcentagem total de sementes germinadas, porcentagem de plântulas normais, o índice de velocidade de germinação (IVG) com base em Maguire (1962), o valor de pico da germinação (VP) com base em Czabator (1962) e a massa de matéria fresca (MF) e seca (MS) de plântulas normais. A massa de matéria fresca das plântulas normais foi determinada em balança analítica com precisão de $0,001 \mathrm{~g}$, e a massa de matéria seca foi avaliada após submeter as plântulas normais à secagem em estufa de circulação de ar a $70^{\circ} \mathrm{C}$ por $48 \mathrm{~h}$.

Os dados das características avaliadas no teste de germinação foram submetidos à análise de variância, segundo o delineamento inteiramente casualizado, com cinco repetições de 20 sementes. Os dados de porcentagem de germinação e de plântulas normais foram submetidos ao teste de normalidade de Liliefors (CRUZ, 2001), e, por não apresentarem distribuição normal, foram transformados em $\operatorname{arcsen}(\mathrm{X} / 100)^{0,5}$. As médias de tratamentos (matrizes) foram comparadas pelo teste de Scott-Knott (SCOTTe KNOTT, 1974) a 5\% de probabilidade.

Os dados de condutividade elétrica e do teste de germinação foram submetidos à análise de correlação de Pearson e a significância testada pelo teste t a 5\% de probabilidade, com o auxílio do programa GENES (CRUZ, 2001). Adicionalmente procedeu-se ao ajuste de regressão polinomial dos valores de germinação (protrusão da radícula) e de plântulas normais em função dos valores de condutividade elétrica das arvores matrizes nos diferentes períodos de embebição. A equação escolhida correspondeu àquela de maior grau com significância estatística $(\mathrm{P} \leq 0,05)$ de seus coeficientes. Esta analise foi processada no programa ESTAT da UNESPJaboticabal.

\section{RESULTADOS E DISCUSSÃO}

Houve variação significativa entre as matrizes em relação à Germinação $(\mathrm{G})$, porcentagem de Plântulas Normais (PN), Índice de Velocidade de Germinação (IVG), Valor Pico da Germinação (VP) e Matéria Fresca (MF) e Seca (MS) de plântulas normais. De modo geral, as matrizes com maiores valores dessas variáveis foram 4 e 5 e as de menores valores, 13, 14, 15 e 16 (Tabela 1). Houve diferenças significativas $(\mathrm{P} \leq 0,01)$ em todas as características avaliadas, o que confirma a alta variabilidade entre as matrizes estudadas.

O IVG variou de 1,05 (M13) a 8,47 (M4), com uma média de 4,11. Foram formados quatro grupos, sendo o primeiro constituído apenas pela matriz 4 (M4), com média de 8,47 ; o segundo grupo é formado por quatro matrizes e variou de 5,37 (M8 e M9) a 6,34 (M10); nove matrizes com IVG variando de 2,66 (M14) a 3,09 (M2) formam o terceiro grupo; e o quarto grupo formouse pelas matrizes M13 (1,05) e M15 (1,92).

Comparando os resultados de IVG e VP, podese considerar que, pelo menos neste caso, o IVG foi mais eficiente na caracterização da qualidade fisiológica das sementes e na discriminação das matrizes, o que pode ser atribuído ao menor CV $(22,95 \%)$ no IVG examinado para esse índice, comparativamente ao observado no VP $(\mathrm{CV}=31,72)$.

O valor pico da germinação (VP) apresentou média de 16,0 com variação de 3,5 (M13) a 40,0 (M4). Foram formados quatro grupos, em que no primeiro está alocada a matriz 4 com VP 40,0 (M4); no segundo grupo, composto por quatro matrizes, a variação foi de 21,5 (M9) a 24,0 (M5); o terceiro grupo foi composto por sete matrizes, com VP variando de 11,2 (M3) a 14,5 (M16); e o quarto e último grupo foi formado por quatro matrizes com VP variando de de 3,5 (M13) a 8,98 (M2).

R. Árvore, Viçosa-MG, v.33, n.4, p.625-634, 2009 
Tabela 1 - Teor de água (TA \%), porcentagem de sementes com protrusão de radícula de radícula (média \pm desvio-padrão) (G) e de plântulas normais (PN), índice de velocidade de germinação (IVG), valor pico (VP), massa de matéria fresca (MF) e matéria seca (MS) de plântulas provenientes de sementes de 16 matrizes de Albizia hassleri (Chodat) Burkart

Table 1 - Water content $(T A \%)$, radicle protusion percentage (mean \pm standard deviation) $(G)$ and normal seedlings percentage $(P N)$, speed of germination index (IVG), peak value (VP), fresh $(M F)$ and dry matter seedlings $(M S)$, obtained from 16 mother trees of Albizia hassleri (Chod.) Burkart

\begin{tabular}{cccccccc}
\hline Fontes de Variação & \multicolumn{7}{c}{ Quadrados Médios } \\
\cline { 2 - 8 } & TA & $\mathrm{G}$ & $\mathrm{PN}$ & $\mathrm{IVG}$ & $\mathrm{VP}$ & $\mathrm{MF}$ & MS \\
\hline Matrizes & - & $643,71^{* *}$ & $947,51^{* *}$ & $17,39^{* *}$ & $402,95^{* *}$ & $0,24^{* *}$ & $0,37^{* * *}$ \\
Erro & - & 59,9 & 71,11 & 0,94 & 24,421 & 0,25 & 0,58 \\
\hline CV $(\%)$ & - & 14,58 & 26,24 & 22,95 & 31,72 & 37,66 & 36,91 \\
\hline Médias & - & 53 & 32 & 4,11 & 16 & 0,42 & 0,065 \\
\hline Matrizes & & & & Médias & & & \\
\hline 1 & 10,5 & $51,0(60 \pm 11,7) \mathrm{c}$ & $39,0(41) \mathrm{b}$ & $3,40 \mathrm{c}$ & $12,5 \mathrm{c}$ & $0,65 \mathrm{~b}$ & $0,074 \mathrm{~b}$ \\
2 & 10,9 & $55,8(68 \pm 5,7) \mathrm{b}$ & $36,6(36) \mathrm{b}$ & $3,09 \mathrm{c}$ & $8,98 \mathrm{~d}$ & $0,49 \mathrm{c}$ & $0,067 \mathrm{~b}$ \\
3 & 11,0 & $56,4(69 \pm 11,4) \mathrm{b}$ & $41,4(44) \mathrm{b}$ & $3,42 \mathrm{c}$ & $11,2 \mathrm{c}$ & $0,60 \mathrm{~b}$ & $0,069 \mathrm{~b}$ \\
4 & 12,2 & $73,8(90 \pm 7,9) \mathrm{a}$ & $54,0(65) \mathrm{a}$ & $8,47 \mathrm{a}$ & $40,0 \mathrm{a}$ & $0,65 \mathrm{~b}$ & $0,079 \mathrm{~b}$ \\
5 & 12,0 & $65,2(82 \pm 6,7) \mathrm{a}$ & $51,6(61) \mathrm{a}$ & $6,50 \mathrm{~b}$ & $24,0 \mathrm{~b}$ & $0,85 \mathrm{a}$ & $0,120 \mathrm{a}$ \\
6 & 14,7 & $44,4(49 \pm 13,8) \mathrm{c}$ & $30,0(25) \mathrm{c}$ & $3,65 \mathrm{c}$ & $13,3 \mathrm{c}$ & $0,29 \mathrm{~d}$ & $0,051 \mathrm{~b}$ \\
7 & 12,7 & $49,8(58 \pm 14,4) \mathrm{c}$ & $30,6(26) \mathrm{c}$ & $3,41 \mathrm{c}$ & $11,3 \mathrm{c}$ & $0,39 \mathrm{c}$ & $0,072 \mathrm{~b}$ \\
8 & 11,3 & $55,4(67 \pm 15,2) \mathrm{b}$ & $37,2(37) \mathrm{b}$ & $5,37 \mathrm{~b}$ & $22,0 \mathrm{~b}$ & $0,51 \mathrm{c}$ & $0,070 \mathrm{~b}$ \\
9 & 11,6 & $66,2(80 \pm 14,6) \mathrm{a}$ & $39,0(40) \mathrm{b}$ & $5,37 \mathrm{~b}$ & $21,5 \mathrm{~b}$ & $0,41 \mathrm{c}$ & $0,068 \mathrm{~b}$ \\
10 & 13,0 & $61,2(77 \pm 4,4) \mathrm{a}$ & $40,2(42) \mathrm{b}$ & $6,34 \mathrm{~b}$ & $23,8 \mathrm{~b}$ & $0,46 \mathrm{c}$ & $0,063 \mathrm{~b}$ \\
11 & 11,6 & $46,2(52 \pm 8,3) \mathrm{c}$ & $25,0(18) \mathrm{c}$ & $3,81 \mathrm{c}$ & $14,4 \mathrm{c}$ & $0,28 \mathrm{~d}$ & $0,069 \mathrm{~b}$ \\
12 & 9,2 & $59,6(74 \pm 7,4) \mathrm{b}$ & $34,8(34) \mathrm{b}$ & $4,25 \mathrm{c}$ & $14,3 \mathrm{c}$ & $0,49 \mathrm{c}$ & $0,088 \mathrm{~b}$ \\
13 & 12,6 & $26,0(19 \pm 4,1) \mathrm{d}$ & $8,80(4,0) \mathrm{d}$ & $1,05 \mathrm{~d}$ & $3,5 \mathrm{~d}$ & $0,10 \mathrm{e}$ & $0,023 \mathrm{c}$ \\
14 & 15,3 & $47,4(54 \pm 17,8) \mathrm{c}$ & $3,60(2,0) \mathrm{d}$ & $2,66 \mathrm{c}$ & $7,9 \mathrm{~d}$ & $0,15 \mathrm{e}$ & $0,032 \mathrm{c}$ \\
15 & 10,5 & $15,0(44 \pm 13,8) \mathrm{c}$ & $25,2(20) \mathrm{c}$ & $1,92 \mathrm{~d}$ & $5,9 \mathrm{~d}$ & $0,36 \mathrm{c}$ & $0,081 \mathrm{~b}$ \\
16 & 10,0 & $49,8(58 \pm 7,6) \mathrm{c}$ & $17,2(16) \mathrm{d}$ & $3,60 \mathrm{c}$ & $14,5 \mathrm{c}$ & $0,16 \mathrm{e}$ & $0,036 \mathrm{c}$ \\
\hline
\end{tabular}

Médias seguidas por uma mesma letra não diferem pelo teste de Scott-Knott (P>0,05). Valores entre parênteses referem aos dados não transformados. $* *=$ Significativo $(\mathrm{P} \leq 0,01)$ pelo teste $\mathrm{F}$.

O VP foi proposto por Czabator (1962), especificamente para sementes florestais, e representa o máximo da razão entre a porcentagem de germinação acumulada e o número de dias decorrido da instalação do teste para se obtê-la. Para Bonner (1998), esse é um dos índices que melhor se presta para avaliar a qualidade de diferentes lotes de sementes. No Brasil, o uso desse índice é limitado. O mesmo fora usado por Botezelli et al. (2000) para obter o valor de germinação na caracterização de quatro procedências de Dipteryx alata (baru). Aguiar (1984) apresentou diferentes índices para avaliação da qualidade fisiológica de sementes florestais, entre os quais se encontra o VP. Silva e Nakagawa (1995), estudando fórmulas para o cálculo da velocidade de germinação, referiram-se ao VP como a germinação instantânea, pois esse é obtido pela derivação da curva de germinação acumulada em relação ao tempo, sendo, portanto, o ponto de inflexão da curva sigmoide que descreve o processo germinativo. De acordo com esses últimos autores, o VP contempla tanto a quantidade de sementes germinadas quanto o tempo demandado para a germinação.
Neste trabalho, o VP demonstrou-se menos sensível na avaliação da qualidade das sementes das diferentes matrizes do que $\mathrm{G}$ e PN, identificando menor número de grupos que esses dois caracteres.

Além da variabilidade na germinação entre matrizes observadas neste trabalho, outros fatores contribuem, de forma expressiva, para a variabilidade dos resultados das pesquisas com sementes florestais, a exemplo do efeito de procedências. Nesse sentido, Alves et al. (2005), trabalhando com sementes de Mimosa caesalpiniifolia, verificaram que a germinação não foi influenciada pelo tamanho das sementes, no entanto essa foi fortemente influenciada pela procedência. $\mathrm{O}$ vigor das sementes apresentou relação direta com o seu tamanho, justificando-se a adoção de classes de tamanho para a formação de mudas.

Os valores de coeficientes de variação experimental (Tabela 1) variaram de médios a muito altos, conforme a classificação de Gomes (2000), mas são compatíveis com resultados de experimentos com sementes de espécies florestais nativas não melhoradas geneticamente. 
Isso indica grande heterogeneidade nos lotes de sementes empregados, os quais, muitas vezes, são caracterizados por grande variação em tamanho e massa de sementes.

As matrizes 4, 5, 9 e 10 superaram as demais quanto à germinação, com valores variando de 77 a 90\%; as matrizes 2, 3, 8 e 12 não diferiram entre si e apresentaram germinação entre 67 e 74\%; as matrizes $1,6,7,11,14$, 15 e 16 mostraram germinação entre 44 e $60 \%$ e não diferiram entre si; e a matriz 13 teve a menor germinação (19\%) entre as 16 matrizes estudadas.

Houve variação quanto à condutividade elétrica (CE) entre as matrizes (Tabela 2) em todos os períodos avaliados, e os coeficientes de variação oscilaram entre médios (10 a 20\%) a muito altos (superior a 30\%), conforme classificação de Gomes (2000).

Verificou-se que a matriz 2 apresentou os menores valores de $\mathrm{CE}$ em todos os períodos de embebição. Até $12 \mathrm{~h}$ de embebição, a matriz 14 foi a que liberou maior quantidade de lixiviados, sendo, contudo, superada pela matriz 13 a partir de $24 \mathrm{~h}$. A quantidade de exsudatos lixiviados das sementes, na água de embebição, pode ser influenciada pelo grau de deterioração, pelo estádio de desenvolvimento no momento da colheita e pela incidência de danos causados pela velocidade de embebição (LOEFFLER, 1981), pela temperatura e tempo de embebição (POWELL, 1986) e por injúrias no tegumento da semente. Notou-se ainda que, mesmo com menor valor de CE, a matriz 2 não aparece entre as de melhor desempenho no teste de germinação (Tabela 1), o que normalmente não é esperado. O que se espera é que, nas condições de baixa germinação, os valores de condutividade sejam altos, como o verificado com a matriz 13.

Fanti e Perez (2005) observaram que o decréscimo no vigor de sementes de Chorisia speciosa (paineira), envelhecidas artificialmente, foi diretamente proporcional ao aumento da lixiviação eletrolítica dos solutos celulares das sementes. Santos (2004) verificou que ambos os testes, de condutividade elétrica e de germinação, possibilitaram igualmente a discriminação dos lotes de sementes de Sebastiana commersoniana. Isso não ocorreu nesta pesquisa.

Tabela 2 - Condutividade elétrica $\left(\mu \mathrm{S} \mathrm{cm}^{-1} \mathrm{~g}^{-1}\right)$ dos lotes de sementes de 16 matrizes de Albizia hassleri (Chodat) Burkart. embebidas em $75 \mathrm{~mL}$ de água destilada, por diferentes períodos a $25^{\circ} \mathrm{C}$

Table 2 - Electrical conductivity $\left(\mu \mathrm{cm}^{-1} \mathrm{~g}^{-1}\right)$ of the seed lots from 16 mother trees of Albizia hassleri (Chodat) Burkart. soaked in $75 \mathrm{~mL}$ of distilled water during different periods at $25^{\circ} \mathrm{C}$

\begin{tabular}{|c|c|c|c|c|c|c|c|c|c|}
\hline \multirow{2}{*}{$\begin{array}{l}\text { Fontes de } \\
\text { Variação }\end{array}$} & \multicolumn{9}{|c|}{ Quadrados Médios } \\
\hline & CE2h & $\mathrm{CE} 4 \mathrm{~h}$ & CE6h & $\mathrm{CE} 12 \mathrm{~h}$ & CE24h & CE48h & CE72h & CE96h & CE120h \\
\hline Matrizes & $9560,6 * *$ & $26677,4 * *$ & $31940,6^{* *}$ & $31940,6^{* * *}$ & $54816,7 * *$ & $87385,5 * *$ & $205642,7 * *$ & $2470,9 * *$ & $271880,2 * *$ \\
\hline Erro & 396,5 & 649,5 & 751,4 & 751,4 & 929,2 & 3051,4 & 8110,3 & 8849,4 & 8669,5 \\
\hline $\mathrm{CV}$ & 41,31 & 28,9 & 25,5 & 22,2 & 16,8 & 23,8 & 31,4 & 29,08 & 26,26 \\
\hline Media & 48,2 & 88,0 & 107,3 & 140,1 & 181,6 & 231,60 & 286,8 & 323,5 & 354,3 \\
\hline \multirow[t]{2}{*}{ Matrizes } & \multicolumn{9}{|c|}{ Médias em diferentes períodos de embebição (horas) } \\
\hline & 2 & 4 & 6 & 12 & 24 & 48 & 72 & 96 & 120 \\
\hline 1 & $17,3 \mathrm{a}$ & $23,2 \mathrm{a}$ & $31,4 \mathrm{a}$ & $47,9 \mathrm{a}$ & $87,4 b$ & $112,0 \mathrm{a}$ & $123,5 \mathrm{a}$ & $139,1 \mathrm{a}$ & $162,5 \mathrm{a}$ \\
\hline 2 & $14,3 \mathrm{a}$ & $20,4 a$ & $28,5 \mathrm{a}$ & $39,6 a$ & $46,5 \mathrm{a}$ & $50,9 \mathrm{a}$ & $79,9 \mathrm{a}$ & $107,6 \mathrm{a}$ & $124,1 \mathrm{a}$ \\
\hline 3 & $16,4 \mathrm{a}$ & $32,2 \mathrm{a}$ & $40,1 \mathrm{a}$ & $51,7 \mathrm{a}$ & $62,3 a$ & $83,6 a$ & $91,4 a$ & $107,9 \mathrm{a}$ & $126,3 \mathrm{a}$ \\
\hline 4 & $36,8 b$ & $56,2 \mathrm{a}$ & $71,1 b$ & $123,2 b$ & $195,4 d$ & $235,4 b$ & $280,5 b$ & $306,4 b$ & $334,2 b$ \\
\hline 5 & $20,1 \mathrm{a}$ & $51,8 \mathrm{a}$ & $71,2 b$ & $112,1 b$ & $141,6 c$ & $204,0 b$ & $226,5 b$ & $243,2 b$ & $262,4 a$ \\
\hline 6 & $104,5 \mathrm{c}$ & $215,7 \mathrm{c}$ & $237,7 d$ & $281,7 \mathrm{c}$ & $318,8 \mathrm{e}$ & $377,4 d$ & $517,8 \mathrm{c}$ & $528,4 \mathrm{c}$ & $576,4 \mathrm{c}$ \\
\hline 7 & $40,3 b$ & $95,7 b$ & $124,6 \mathrm{c}$ & $161,3 b$ & $215,4 d$ & $290,3 \mathrm{c}$ & $253,9 b$ & $350,5 b$ & $392,5 b$ \\
\hline 8 & $41,9 b$ & $89,5 b$ & $121,4 \mathrm{c}$ & $154,8 b$ & $199,4 d$ & $219,6 b$ & $253,8 b$ & $270,7 b$ & $293,9 b$ \\
\hline 9 & $23,4 a$ & $50,9 a$ & $78,7 \mathrm{c}$ & $125,5 b$ & $160,7 \mathrm{c}$ & $197,8 b$ & $246,1 b$ & $281,2 b$ & $319,2 b$ \\
\hline 10 & $38,2 b$ & $77,7 b$ & $105,2 \mathrm{c}$ & $142,7 b$ & $191,5 d$ & $236,1 b$ & $279,3 b$ & $314,5 b$ & $335,8 b$ \\
\hline 11 & $44,9 b$ & $84,2 b$ & $105,6 \mathrm{c}$ & $137,9 b$ & $205,4 d$ & $280,4 \mathrm{c}$ & $348,1 b$ & $391,5 b$ & $421,6 b$ \\
\hline 12 & $16,4 \mathrm{a}$ & $26,8 \mathrm{a}$ & $35,7 a$ & $54,4 \mathrm{a}$ & $88,5 b$ & $120,1 \mathrm{a}$ & $147,5 \mathrm{a}$ & $185,1 \mathrm{a}$ & $210,9 a$ \\
\hline 13 & $97,1 \mathrm{c}$ & $193,8 \mathrm{c}$ & $226,9 d$ & $293,7 \mathrm{c}$ & $401,5 f$ & $553,5 \mathrm{e}$ & $881,6 d$ & $988,4 d$ & $1050,5 d$ \\
\hline 14 & $178,5 d$ & $263,8 d$ & $296,4 \mathrm{e}$ & $336,4 d$ & $364,7 f$ & $421,9 d$ & $501,8 \mathrm{c}$ & $569,6 \mathrm{c}$ & $608,7 \mathrm{c}$ \\
\hline 15 & $49,6 b$ & $82,6 b$ & $88,6 c$ & $107,7 b$ & $125,0 b$ & $194,9 b$ & $210,2 b$ & $221,1 \mathrm{a}$ & $249,9 a$ \\
\hline 16 & $31,7 b$ & $44,5 \mathrm{a}$ & $54,0 \mathrm{~b}$ & $71,8 \mathrm{a}$ & $103,1 b$ & $128,7 \mathrm{a}$ & $147,8 \mathrm{a}$ & $171,7 \mathrm{a}$ & $200,5 a$ \\
\hline
\end{tabular}

** Significativo $(\mathrm{P} \leq 0,01)$ pelo teste F. Médias seguidas da mesma letra na coluna não diferem entre si, pelo teste de ScottKnott ( $\mathrm{P}>0,05)$. 
No início do experimento de CE, as sementes apresentavam teores de água variando de 9,2\% (matriz 12) a $15,3 \%$ (matriz 14). Marques (2001) verificou que a variação do teor de água das sementes de Dalbergia nigra de 9,4 a 10,6\% não interferiu nos resultados dos testes de CE.

Bonner (1986) verificou que, em sementes de Pinus elliottii e P. taeda, apenas os testes de tetrazólio e condutividade elétrica apresentaram correlação com a germinação e que o teste de condutividade elétrica é promissor, já que fornece resultados mais rápidos do que o teste de germinação.

Estudos de aplicação do teste de condutividade elétrica em sementes florestais vêm sendo realizados com a finalidade de se adaptar a metodologia aos diferentes tipos de espécies arbóreas, como coníferas, folhosas e grupos sucessionais (pioneiras, secundárias e climácicas). Barbedo e Cícero (1998) verificaram que o teste de condutividade elétrica foi promissor para diferenciar lotes de sementes de Inga uruguensis em três categorias de germinação, pois ambos os testes, de CE e germinação, apresentaram correlação. Marques et al. (2002a, b) observaram que o uso do teste de condutividade elétrica apresentou resultados satisfatórios para a avaliação da qualidade fisiologia de três lotes de sementes de Dalbergia nigra. Esse teste também foi promissor para diferenciar a qualidade fisiológica de diferentes lotes de sementes de Sebastiania commersoniana (SANTOS e PAULA, 2005).

Aguiar et al. (2001), estudando a variabilidade entre 30 matrizes de polinização livre de Astronium fraxinifolium, concluíram ser a condutividade elétrica a característica mais indicada, entre outras avaliadas, para a seleção de progênies com sementes de melhor qualidade. Contudo, Torres (2002), trabalhando com sementes de melão, o teste de condutividade elétrica não foi eficiente. Tesser (2005) não encontrou resultados satisfatórios desse teste para a avaliação do vigor de lotes de sementes de Solanum lycocarpum e $S$. granulosum-leprosum, embora para $S$. pseudoquina essa autora tenha obtido resultados promissores, relacionados ao teste de germinação. Essa mesma autora atribuiu à grande variabilidade genética dos lotes de sementes de $S$. lycocarpum e $S$. granulosum-leprosum a baixa correlação encontrada entre os resultados do teste de CE e de germinação. Paula (2007), trabalhando com sementes de Pterogyne nitens (amendoim-do-campo), verificou que o teste de condutividade elétrica foi adequado para avaliar a qualidade fisiológica das sementes.

As estimativas dos coeficientes de correlação entre os resultados de condutividade elétrica com os dos testes de germinação (Tabela 3), embora significativos na maioria dos casos, foram de baixa magnitude, evidenciando-se a fraca associação entre eles.

As equações de regressão polinomial obtidas entre a porcentagem de germinação e de plântulas normais das diferentes matrizes, como variável dependente, em cada período de embebição, apresentaram baixos coeficientes de determinação em função dos valores de condutividade elétrica $\left(R^{2}\right.$ entre 0,27 e 0,56), evidenciando-se uma baixa associação entre essas características (Figuras 1 e 2).

Tabela 3 - Coeficientes de correlação linear simples para a porcentagem de germinação (G), porcentagem de plântulas normais (PN), massa de matéria fresca (MF) e matéria seca (MS) de plântulas, valor pico de germinação (VP), índice de velocidade de germinação (IVG), em relação à condutividade elétrica $\left(\mu \mathrm{S} \mathrm{cm}^{-1} \mathrm{~g}^{-1}\right)$ dos lotes de sementes provenientes de 16 matrizes de Albizia hassleri (Chod.) Burkart. embebidas em $75 \mathrm{~mL}$ de água destilada por diferentes períodos de embebição a $25^{\circ} \mathrm{C}$

Table 3 - Linear correlation coefficients between the percentage of germination $(G)$, normal seedling percentage $(P N)$, fresh $(M F)$ and dry matter of seedlings $(M S)$, peak value (VP), speed germination index (IVG), in relation to the electrical conductivity $\left(\mu \mathrm{cm}^{-1} \mathrm{~g}^{-1}\right)$ of the different seed lots of 16 mother trees of Albizia hassleri (Chod.) Burkart. soaked in $75 \mathrm{~mL}$ of distilled water for different soaking periods at $25^{\circ} \mathrm{C}$

\begin{tabular}{|c|c|c|c|c|c|c|c|c|c|}
\hline Variáveis & CE-2h & CE-4h & CE-6h & CE-12h & CE-24h & CE-48h & $\mathrm{CE}-72 \mathrm{~h}$ & CE-96h & $\mathrm{CE}-120 \mathrm{~h}$ \\
\hline $\mathrm{G}$ & $-0,45 * *$ & $-0,54 * *$ & $-0,51 * *$ & $-0,49 * *$ & $-0,48 * *$ & $-0,54 * *$ & $-0,57 * *$ & $-0,60 * *$ & $-0,59 * *$ \\
\hline $\mathrm{PN}$ & $-0,58 * *$ & $-0,60 * *$ & $-0,60 * *$ & $-0,54 * *$ & $-0,52 * *$ & $-0,54 * *$ & $-0,52 * *$ & $-0,55 * *$ & $-0,56 * *$ \\
\hline MF & $-0,56 * *$ & $-0,59 * *$ & $-0,59 * *$ & $-0,56 * *$ & $-5,83 * *$ & $-0,55 * *$ & $-0,54 * *$ & $-0,55 * *$ & $-0,57 * *$ \\
\hline MS & $-0,53 * *$ & $-0,55 * *$ & $-0,54 * *$ & $-0,52 * *$ & $-050 * *$ & $-0,46^{* *}$ & $-0,48 * *$ & $-0,49 * *$ & $-0,49 * *$ \\
\hline VP & $-0,31 * *$ & $-0,33 * *$ & $-0,29 * *$ & $-0,213^{\mathrm{ns}}$ & $-0,14^{\mathrm{ns}}$ & $-0,21^{\mathrm{ns}}$ & $-0,23 *$ & $-2,408 *$ & $-0,26^{*}$ \\
\hline IVG & $-0,35 * *$ & $-0,38 * *$ & $-0,34 * *$ & $-0,27 *$ & $-0,22 *$ & $-0,29 * *$ & $-0,32 * *$ & $-0,36 * *$ & $-0,36 * *$ \\
\hline
\end{tabular}

$* \mathrm{e}^{* *}$ Significativos, respectivamente $(\mathrm{P} \leq 0,05)$ e $(\mathrm{P} \leq 0,01)$. ${ }^{\mathrm{ns}}$ Não significativo $(\mathrm{P}>0,05)$.

R. Árvore, Viçosa-MG, v.33, n.4, p.625-634, 2009 

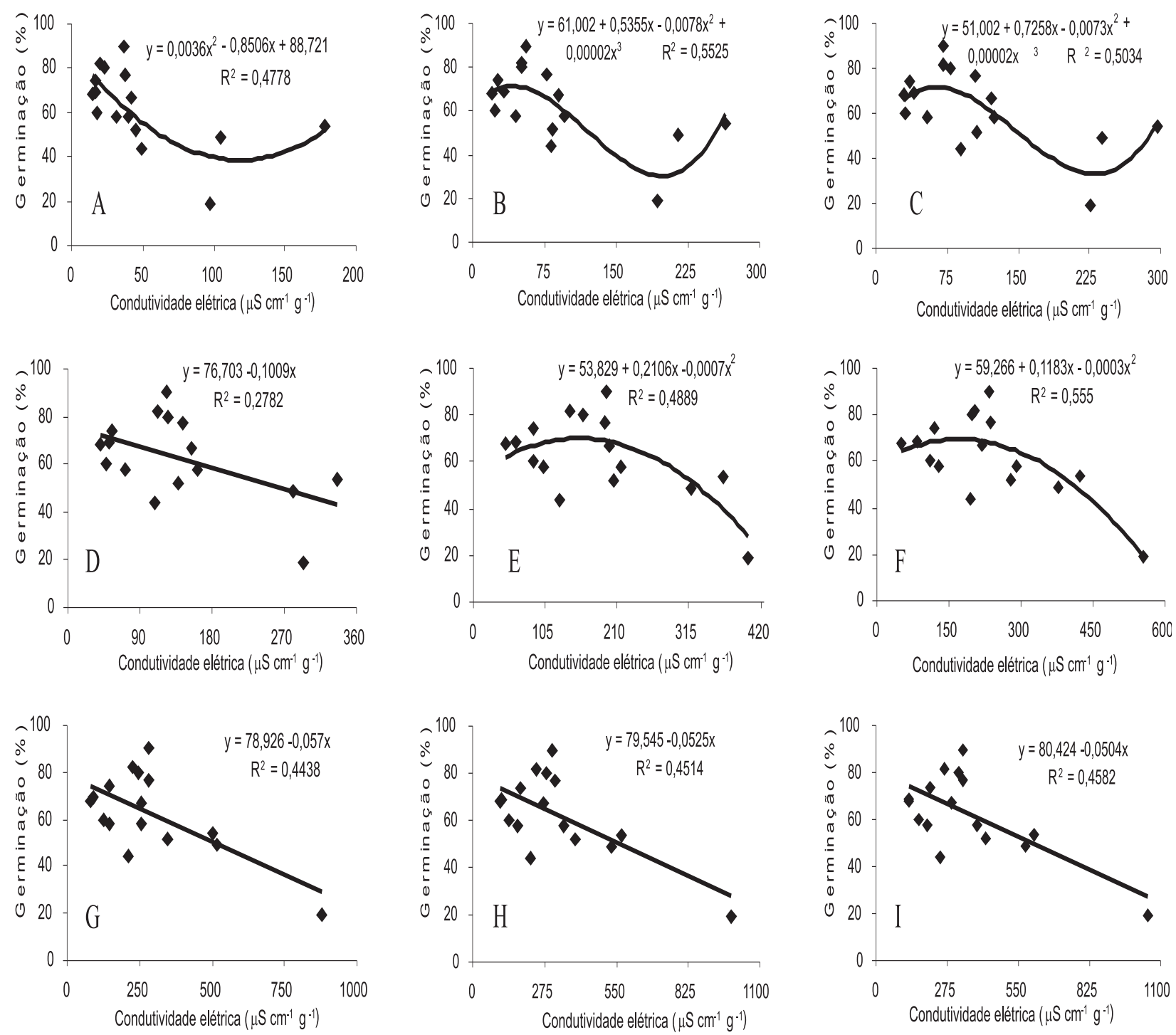

Figura 1 - Germinação (protrusão da radícula) de sementes de Albizia hassleri (Chodat) Burkart. provenientes de diferentes matrizes em função dos valores de condutividade elétrica e diferentes períodos de embebição a $25^{\circ} \mathrm{C}(\mathrm{A}=2 \mathrm{~h})$; $(\mathrm{B}=4 \mathrm{~h})(\mathrm{C}=6 \mathrm{~h}) ;(\mathrm{D}=12 \mathrm{~h}) ;(\mathrm{E}=24 \mathrm{~h}) ;(\mathrm{F}=48 \mathrm{~h}) ;(\mathrm{G}=72 \mathrm{~h}) ;(\mathrm{H}=96 \mathrm{~h}) ; \mathrm{e}(\mathrm{I}=120 \mathrm{~h})$.

Figure 1 - Germination (radicle protrusion) of seeds Albizia hassleri (Chodat) Burkart. of different mother trees according to the values of electrical conductivity at different soaking periods at $25^{\circ} \mathrm{C}(A=2 \mathrm{~h}) ;(B=4)(C=6 \mathrm{~h})$; $(D=12 h) ;(E=24 h) ;(F=48 h) ;(G=72 h) ;(H=96 h) ;(I=120 h)$.

Um dos fatores que pode ter contribuído para esses resultados é a grande variabilidade genética entre os indivíduos, conforme relatado por Bonner (1998). Este considerou que as diferenças genéticas entre os lotes de sementes vindas de populações silvestres estão entre as principais causas de dificuldade de padronização do teste de CE. Isso é especialmente importante em espécies selvagens, sem domesticação e melhoramento. Também, afirmou que o teste de condutividade elétrica em sementes florestais dificilmente terá o mesmo desempenho do que em sementes de grandes culturas, mas que é uma ferramenta que pode auxiliar, em combinação com outros testes, na identificação de lotes de diferentes qualidades fisiológicas.

R. Árvore, Viçosa-MG, v.33, n.4, p.625-634, 2009 

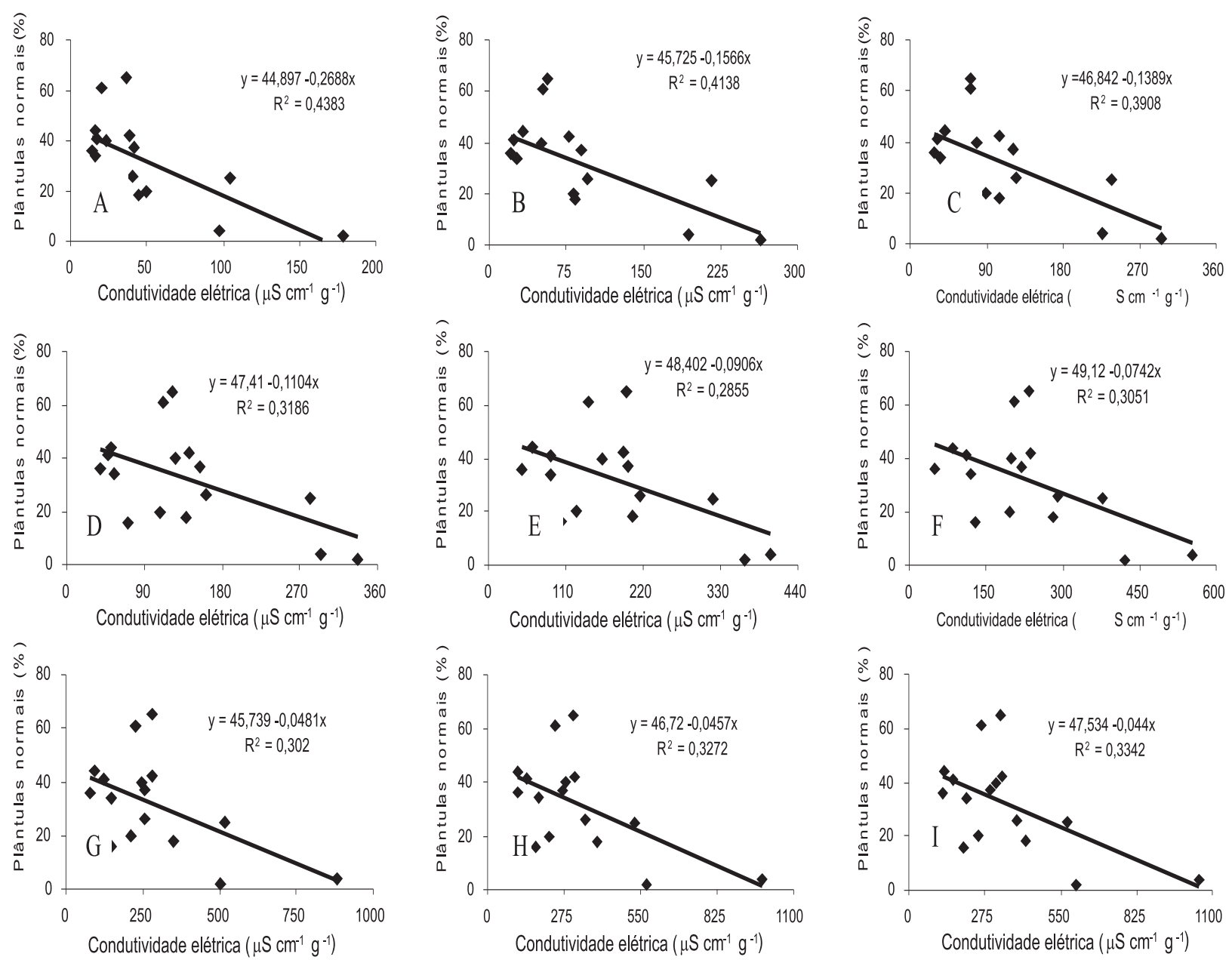

Figura 2 - Plântulas normais resultantes de sementes de Albizia hassleri (Chodat) Burkart. provenientes de diferentes matrizes em função dos valores de condutividade elétrica e diferentes períodos de embebição a $25^{\circ} \mathrm{C}(\mathrm{J}=2 \mathrm{~h})$; $(\mathrm{K}=4 \mathrm{~h})(\mathrm{L}=6 \mathrm{~h}) ;(\mathrm{M}=12 \mathrm{~h}) ;(\mathrm{N}=24 \mathrm{~h}) ;(\mathrm{O}=48 \mathrm{~h}) ;(\mathrm{P}=72 \mathrm{~h}) ;(\mathrm{Q}=96 \mathrm{~h}) ; \mathrm{e}(\mathrm{R}=120 \mathrm{~h})$

Figure 2 - Normal seedlings obtained from seeds of Albizia hassleri (Chodat) Burkart. of different mother trees according to the values of electrical conductivity at different soaking periods at $25^{\circ} \mathrm{C}(\mathrm{J}=2 \mathrm{~h}) ;(K=4 \mathrm{~h})(L=6 \mathrm{~h})$; $(M=12 h) ;(N=24 h) ;(O=48 h) ;(P=72 h) ;(Q=96 h) ;(R=120 h)$.

\section{CONCLUSÃO}

O teste de condutividade, conduzido a $25^{\circ} \mathrm{C}$ por períodos de 2 a 120 h de embebição em $75 \mathrm{~mL}$ de água destilada, não foi eficiente para discriminar as matrizes de Albizia hassleri quanto à qualidade das sementes.

\section{REFERÊNCIAS}

AGUIAR, A. V. et al. Determinação de parâmetros genéticos em população de gonçalo-alves (Astronium fraxinifolium) através das características fisiológicas da semente. Scientia Forestalis, n.60, p.89-97, 2001.
AGUIAR, I. B. Avaliação da qualidade fisiológica das sementes florestais. In: SEMINÁRIO INTERNACIONAL: MÉTODOS DE PRODUÇÃO E CONTROLE DE QUALIDADE DE SEMENTES E MUDAS FLORESTAIS, 1984, Curitiba. Anais... Curitiba: FUPEF, 1984. p.277-290.

ALVES, E. U. et al. Influencia do tamanho e da procedência de sementes de Mimosa caesalpiniifolia Benth. sobre a germinação e vigor. Revista Árvore, v.29, n.3, p.877-885, 2005 .

R. Árvore, Viçosa-MG, v.33, n.4, p.625-634, 2009 
ASSOCIATION OF OFFICIAL SEED ANALYSTS AOSA Seed vigour testing handbook. Lincoln: 1983. 88p. (Handbook on Seed Testing. Contribution, 32).

BARBEDO, C. J.; CÍCERO, S. M. Utilização do teste de condutividade elétrica para previsão do potencial germinativo de sementes de ingá.

Scientia Agrícola, v.55, n.2, p.249-259, 1998.

BONNER, F. T. Measurement of seed vigor for loblolly and slash pines. Forest Science, v.32, n.1, p.170-178, 1986.

BONNER, F. T. Testing tree seeds for vigor: a review. Seed Technology, v.20, n.1, p.5-17, 1998.

BOTEZELLI, L.; DAVIDE, A. C.; MALAVASI, M. M. Características dos frutos e sementes de quatro procedêcnias de Dipteryx alata Vogel. (baru). Cerne, v.6, n.1, p.9-18, 2000.

BRASIL. Ministério da Agricultura e Reforma Agrária. Regras para analise de sementes. Brasília: SNDA/DNDV/CLAV, 1992.356p.

CAPELANES, T. M. C.; BIELLA, L. C. Programa de produção e tecnologia de sementes de espécies florestais nativas desenvolvido pela Companhia Energética de São Paulo - CESP. In: SIMPÓSIO BRASILEIRO SOBRE TECNOLOGIA DE

SEMENTES FLORESTAIS, 1., 1984, Belo Horizonte. Anais... Belo Horizonte: 1984. p.85-107.

CRUZ, C. D. Programa GENES: versão Windows: aplicativo computacional em genética e estatística. Viçosa, MG: Universidade Federal de Viçosa, 2001. 648p.

CZABATOR, F. J. Germination value: an index combining speed and completeness of pine seed germination. Forest Science, v.8, n.4, p. 386-396, 1962.

DIAS, D. C. F. S.; MARCOS FILHO, J. Testes de vigor baseados na permeabilidade das membranas celulares. I. Condutividade elétrica.

Informativo Abrates, v.5, n.1, p.26-36, 1995.

DURAN, J. M.; TORTOSA, M. E. The effect of mechanical and chemical scarification germination of charlock $S$. arvensis. Seed Science $\&$ Technology, v.13, n.1, p.155-163, 1985.
FANTI, S.C.; PEREZ, S.C.J.G.A. Efeitos do envelhecimento precoce no vigor de sementes de Chorisia speciosa St. Hil. - Bombacaceae. Revista Árvore, v.29, n.3, p.345-352, 2005.

FERRAZ, I.D.K.; LIMA, V.N.S.; COSTA, M.M. Teste de viabilidade em sementes de Carapa procera. In: SIMPÓSIO BRASILEIRO SOBRE TECNOLOGIA DE SEMENTES FLORESTAIS, 2., 1991. Atibaia. Anais... São Paulo: Instituto Florestal, 1991. p.39. (Série Documentos).

GOMES, F. P. Curso de estatística experimental. 14.ed. Piracicaba: Ed. F. P. Gomes, 2000. 477p.

HAMPTON, J. G.; TEKRONY, D. M. (EdS.). Handbook of vigour teste methods. 3.ed. Zurich: The International Seed Testing Association, 1995. 117p.

LOEFFLER, T. M.; TEKRONY, D. M.; EGLI, D. B. The bulk conductivity test as an indicator of soybean seed quality. Journal of Seed Tecnology, v.12, n.1, p.37-53, 1988.

LOEFFLER, T. M. The bulk conductivity test as an indicator of soybean seed quality. 1981. 181f. Dissertação (Mestrado em Agronomia) - University of Kentucky, Leington, 1981.

LONGHI, R. A. Livro das árvores: e arvoretas do sul. 2.ed. Porto Alegre: L\&PM, 1995. 176p.

LORENZI, R. Árvores brasileiras: manual de identificação, cultivo de plantas arbóreas nativas do Brasil. Nova Odessa: Plantarum, 1992. 368p.

MAGUIRE, J. D. Speed of germination-aid selection and evaluation for seedling emergence and vigor. Crop Science, v.2, n.1, p.176-177, 1962.

MARQUES, M. A. Teste de condutividade elétrica para avaliação da qualidade fisiológica de sementes de Dalbergia nigra Fr. Allem. (Jacarandá-da bahia). 2001. 74f. Dissertação (Mestrado em Produção e Tecnologia de Sementes) - Faculdade de Ciências Agrárias e Veterinárias, Universidade Estadual Paulista, Jaboticabal, 2001.

R. Árvore, Viçosa-MG, v.33, n.4, p.625-634, 2009 
MARQUES, M. A.; PAULA, R. C.; RODRIGUES, T. J. D. Adequação do teste de condutividade elétrica para determinar a qualidade fisiológica de sementes de jacarandá-da-bahia (Dalbergia nigra (Vell.) Fr. All. ex. Benth). Revista Brasileira de Sementes, v.24, n.1, p.271-278, 2002a.

MARQUES, M. A.; PAULA, R. C.; RODRIGUES, T. J. D. Efeito do número de sementes e do volume de água na condutividade elétrica de sementes de Dalbergia nigra (Vell.) Fr. All. ex. Benth. Revista Brasileira de Sementes, v.24, n.1, p.254-262, 2002b.

PAULA. R. C. Repetibilidade e divergência genética entre matrizes de Pterogyne nitens Tul. (Fabaceae - Caesalpinioideae) por caracteres biométricos de frutos e de sementes e parâmetros da qualidade fisiológica de sementes. 2007. 128f. Tese (LivreDocência em Silvicultura) - Faculdade de Ciências Agrárias e Veterinárias, Universidade Estadual Paulista "Julio de Mesquita Filho", Jaboticabal, 2007.

POWELL, A. A. Cell membranes and seed leachate conductivity in relation to the quality of seed for sowing. Journal Seed Technology, v.10, n.2, p.81-100, 1986.

SANTOS, S. R. G. Qualidade fisiológica e armazenamento de sementes de Sebastiania commersoniana (Baill.) Smith \& Downs. 2004. 95f. Tese (Doutorado em Agronomia - Produção Vegetal) Faculdade de Ciências Agrárias e Veterinárias, Universidade Estadual Paulista, Jaboticabal, 2004.

SANTOS, S. R. G.; PAULA, R. C. Teste de condutividade elétrica para avaliação da qualidade fisiológica de sementes de Sebastiania commersoniana (Bail.) Smith \& Downs (branquilho) - Euphorbiaceae. Revista Brasileira de Sementes, v.27, n.2, p.136-145, 2005.
SCOTT, A.; KNOTT, M. Cluster-analys is method for grouping means in analysis of variance. Biometrics, v. 30, n.2, p.507-512, 1974 .

SILVA, J. B. C.; NAKAGAWA, J. Estudo de fórmulas para cálculo da velocidade de germinação. Informativo ABRATES, v.5, n.1, p.62-73, 1995.

TESSER, S. M. Teste de condutividade elétrica para discriminação de lotes de sementes de três espécies arbóreas do gênero Solanum. 2005. 49f. Dissertação (Mestrado em Agronomia Produção e Tecnologia de Sementes) Faculdade de Ciências Agrárias e Veterinárias, Universidade Estadual Paulista, Jaboticabal, 2005.

TONIN, G. A. et al. Influência da temperatura de condicionamento osmótico na viabilidade e no vigor de sementes de Pterogyne nitens Tull. Revista Brasileira de Sementes, v.27, n.2, p.35-43, 2005.

TORRES, S. B. Métodos para avaliação do potencial fisiológico de sementes de melão. 2002. 120f. Tese (Doutorado em Agronomia - Fitotecnia), Escola Superior de Agricultura "Luiz de Queiroz”, Universidade de São Paulo, Piracicaba, 2002.

VIEIRA, R. D.; KRZYZANOWSKI, F.C. Teste de condutividade elétrica. In: KRZYZANOWSKI, F. C. H.; VIEIRA, R. D.; FRANCA NETO, J. B. (Eds) Vigor de sementes: conceitos e testes. Londrina: ABRATES, 1999. p.4-20. 